\title{
Pulmonary disease in polymyositis/dermatomyositis: a clinicopathological analysis of 65 autopsy cases
}

\author{
S LAKHANPAL, J T LIE, D L CONN, AND W J MARTIN II
}

From the Division of Rheumatology and Internal Medicine, Department of Pathology, and Division of Thoracic Disease, Mayo Clinic and Mayo Graduate School of Medicine, Rochester, Minnesota, USA

SUMmaRY The clinical and autopsy records of 65 patients with either polymositis (24) or dermatomyositis (41) and pulmonary disease were reviewed. Pulmonary symptoms were recorded in 43 of the cases and included dyspnoea in 31 , cough in 23 , and chest pain in six. Interstitial lung disease was noted at autopsy in 27 patients; almost half of these had arthritis. Bronchopneumonia was found in 35 patients, 31 of these had received prednisone. Dysphagia was present in a similar proportion of patients with and without pneumonia. Pulmonary vasculitis was seen in five patients; pulmonary symptoms, arthritis, and raised erythrocyte sedimentation rate were present in four of these cases and all five had associated interstitial lung disease. Other pulmonary manifestations included pulmonary oedema, primary pulmonary malignancy, diffuse alveolar damage, fibrinous pleuritis, pulmonary emboli, and diaphragmatic atrophy. The mean survival after disease onset was 29 months but was much less for those with interstitial lung disease and pulmonary vasculitis.

Key words: interstitial lung disease, pulmonary vasculitis, autopsy in polymyositis/ dermatomyositis.

Rheumatic diseases are systemic disorders affecting various organ systems including lungs. ${ }^{1}$ The first report of interstitial lung disease in a patient with dermatomyositis (DM) appeared in $1956 .^{2}$ It has been previously suggested that interstitial pneumonitis, aspiration pneumonia, and ventilatory insufficiency are the three types of pulmonary involvement in polymyositis (PM)/dermatomyositis. ${ }^{3}$ Pulmonary involvement by other pathogenetic mechanisms such as opportunistic infections and iatrogenic disease is possible, however. ${ }^{45}$

Pulmonary disease in an autopsy series has been described in other rheumatic diseases. ${ }^{67}$ We report the cases of 65 patients with polymyositis/ dermatomyositis who had histologically recorded pulmonary disease at autopsy. A variety of disorder was seen, including interstitial lung disease (ILD), pneumonia, pulmonary oedema, fibrinous pleuritis, diaphragmatic atrophy, vasculitis, and pulmonary artery atherosclerosis.

\section{Patients and methods}

The autopsy records of the Mayo Clinic from 1931

Accepted for publication 6 June 1986.

Correspondence to Dr J T Lie, Department of Pathology, Mayo Clinic, Rochester, Minnesota 55905, USA. to 1983 were reviewed, and 88 cases coded as PM/DM with inflammatory myopathy and pulmonary disease were identified. Their clinical records were reviewed, and 65 cases fulfilled the criteria of Bohan and Peter for the diagnosis of PM/DM. ${ }^{8}$ Eight other patients were found to have scleroderma, there was polyarteritis nodosa in four, systemic lupus erythematosus in two, rheumatoid arthritis in one, and myasthenia gravis in one. Seven patients were also excluded because they were below 16 years of age.

Data were abstracted from patients' clinical records on data collection forms and analysed. Autopsy data were similarly abstracted on data collection forms. All histopathology slides were reviewed by one of us (JTL).

\section{Results}

Of the 65 patients, 24 had PM and 41 had DM. There were 24 men and 41 women with a mean and median age at the time of death of 57 years (range 19-86). Of the 24 men, eight had PM and $16 \mathrm{DM}$; the 41 women were divided into 16 with PM and 25 with DM. Pulmonary symptoms had been recorded in 43 of the cases and included dyspnoea in 31 , cough in 23, and chest pain in six (Table 1). 
Auscultatory signs included audible rales in 27 , pericardial rub in two, and pleural rub in one. Dysphagia was present in 34 patients and arthritis in 22. Only eight patients (PM 4, DM 4) were current smokers, and five others were ex-smokers, having stopped 10, 12, 20, 30 years ago and for an unknown period respectively.

Fifty four of the 65 patients were treated with corticosteroids, and seven patients had received additional immunosuppressive agents which included azathioprine (three), cyclophosphamide (two), methotrexate (one), and D-penicillamine (one). Eleven patients had not received any immunosuppressive drugs including corticosteroid preparations; eight of these died between 1931 and 1946 and the other three in 1960, 1966, and 1974 respectively.
Table 1 Clinical features of 65 patients with PM/DM

\begin{tabular}{|c|c|c|c|}
\hline Clinical features & $\begin{array}{l}P M \\
(n)\end{array}$ & $\begin{array}{l}D M \\
(n)\end{array}$ & $\begin{array}{l}\text { Tota } \\
(n)\end{array}$ \\
\hline Dyspnoea & 12 & 19 & 31 \\
\hline Cough & 11 & 12 & 23 \\
\hline Chest pain & 3 & 3 & 6 \\
\hline Rales & 8 & 19 & 27 \\
\hline Pericardial rub & 0 & 2 & 2 \\
\hline Pleural rub & 0 & 1 & 1 \\
\hline Dysphagia & 13 & 21 & 34 \\
\hline Arthritis & 10 & 12 & 22 \\
\hline Current smokers & 4 & 4 & 8 \\
\hline $\begin{array}{l}\text { Mean duration of disease } \\
\text { in months (range) }\end{array}$ & $41(1-264)$ & $22(1-168)$ & 29 \\
\hline $\begin{array}{l}\text { Mean survival after } \\
\text { pulmonary symptoms } \\
\text { in months (range) }\end{array}$ & $10(1-30)$ & $7(0 \cdot 5-24)$ & 8 \\
\hline
\end{tabular}

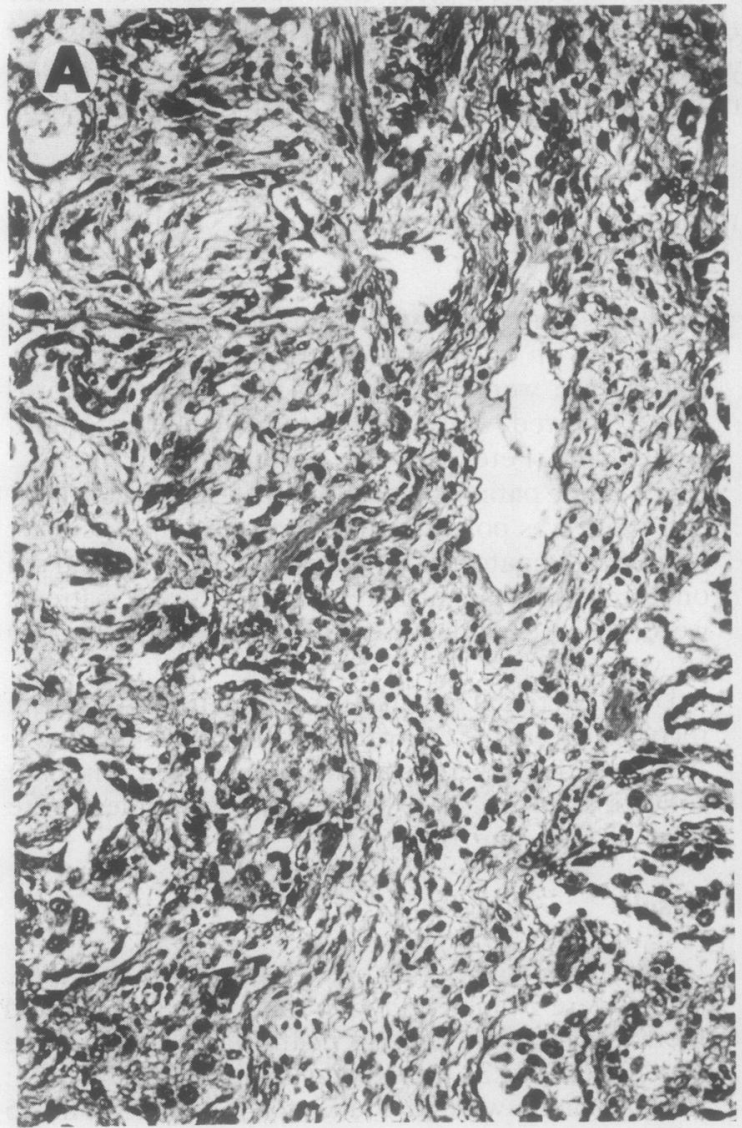

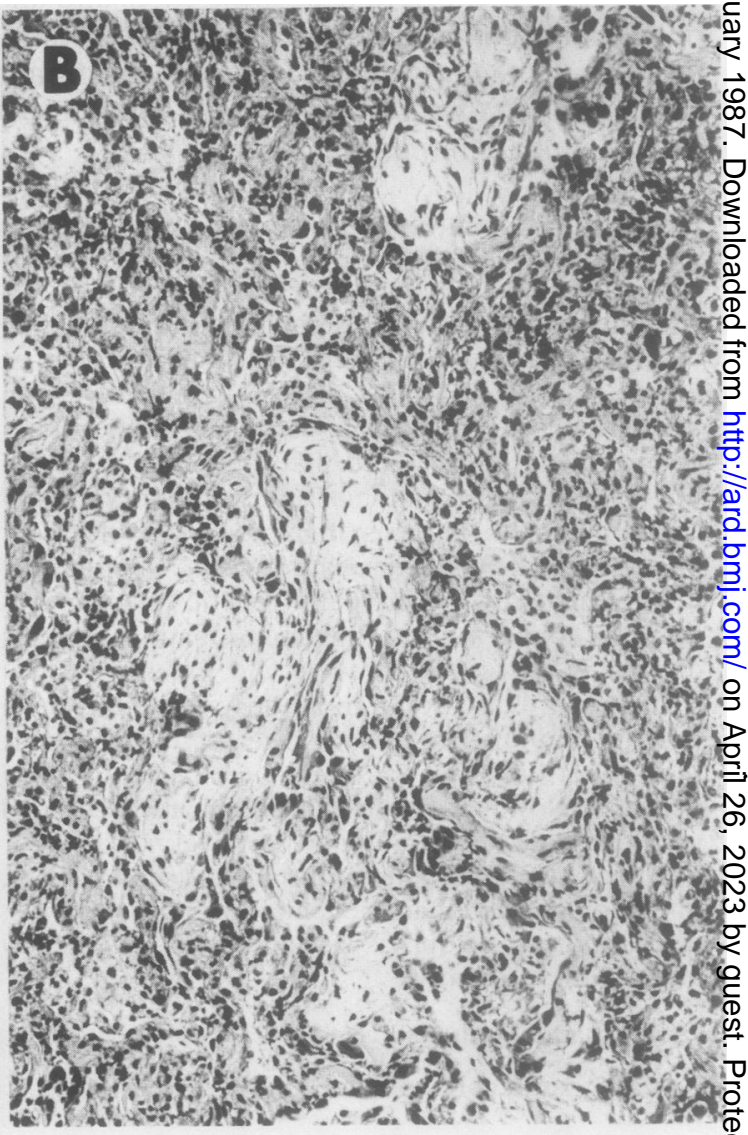

Fig. 1 Histopathological variations of interstitial lung disease in patients with $P M / D M:(A)$ sclerosing alveolitis with hyperplasia of type II pneumocytes and early septal fibrosis; $(B)$ progressive intraluminal and interstitial fibrosis of the bronchoalveolar units of the lung with a moderate lymphomononuclear inflammatory infiltrate. (Haematoxylin and eosin). 
The mean duration from disease onset to death was 29 months but was significantly shortened from onset of pulmonary symptoms to eight months (Table 1).

\section{LA B ORATORY FIN DINGS}

Haemoglobin was below the normal range in 25 cases (PM 4, DM 21), and the erythrocyte sedimentation rate was raised in 42 (PM 15, DM 27). Rheumatoid factor was reactive in three cases and antinuclear antibody positive in three cases. Barium studies of the oesophagus showed changes of PM/ DM in 10 of 31 tested: including absent (three) or feeble (four) oesophageal peristalsis and weak pharyngeal contraction (three). Pulmonary function studies had been performed in only 11 cases and showed restrictive changes in eight and mild airway obstruction in one; the latter patients had over 50 pack years' history of smoking. Lung biopsy had been performed in eight cases (four open and four transbronchial). Seven had changes of ILD and one showed squamous metaplasia. None of the other laboratory test results were found to be clinically significant.

\section{PATHOLOGICAL FINDINGS}

\section{Interstitial lung disease}

ILD was present in 27 patients; 11 had PM and 16 had DM. A radiographic diagnosis of ILD had been made in 18 of the 25 patients and confirmed by lung biopsy in seven (open biopsy in four and transbronchial in three). One patient did not show ILD on transbronchial biopsy but had evidence of ILD at autopsy. Of the 27 patients with evidence of ILD at autopsy, 21 had pulmonary symptoms, including cough, dyspnoea, or chest pain. Arthritis was present in 13 of these 27 cases (48\%) as compared with only nine of the remaining 38 cases $(24 \%)$. None of the patients with ILD had received drugs that might have been causative. The mean survival of patients with ILD after onset of PM/DM was 18 months (PM 31 months, DM 11 months) and reduced to only eight months (PM 14 months, DM 4 months) after onset of pulmonary symptoms. This compared with a mean survival after disease onset of 36 months (PM 50 months, DM 28 months) for patients without ILD.

There were no unique morphological features that distinguished diffuse ILD of PM/DM from that of other connective tissue diseases or from that of idiopathic pulmonary fibrosis. ${ }^{15-7}$ 4-17 The lesions were, as a rule, irregularly distributed within the lung, so that apparently normal areas intermingled with areas of ILD. The histopathological spectrum of ILD in PM/DM ranged from sclerosing alveolitis associated with hyperplasia of type II pneumocytes (Fig. 1A) to progressive intraluminal and interstitial fibrosis of the bronchoalveolar units of the lung (Fig. 1B). The inflammatory infiltrate consisted of predominantly lymphomononuclear and plasma cells; eosinophils and polymorphonuclear cells were seen only rarely.

\section{Pulmonary vasculitis}

Active and healed pulmonary vasculitis was seen in five patients, of whom four had PM and one had DM. Three of these were women and two men, with ages ranging from 40 to 73 years (Table 2). Pulmonary symptoms were present in four (dyspnoea in two, cough in two), and rales were audible in four. Four of the five patients had associated arthritis of the hands with involvement of the wrists and knees in two each, and elbows, shoulders, and ankles in one each. The ESR was significantly raised in four cases but was $1 \mathrm{~mm} / 1 \mathrm{st}$ hour in one case. The latter patient, whose case has previously been reported, ${ }^{18}$ had significant pulmonary hypertension with polycythaemia, which might have contributed to the lower ESR.

Interestingly, all patients with pulmonary vasculitis also had ILD, and one had evidence of vasculitis on muscle biopsy. Histopathologically, the pulmonary vasculitis in PM/DM was indistinguishable from that observed in other connective tissue diseases, such as systemic lupus erythematosus, scleroderma,

Table 2 Clinical and laboratory features of patients with PM/DM and pulmonary vasculitis

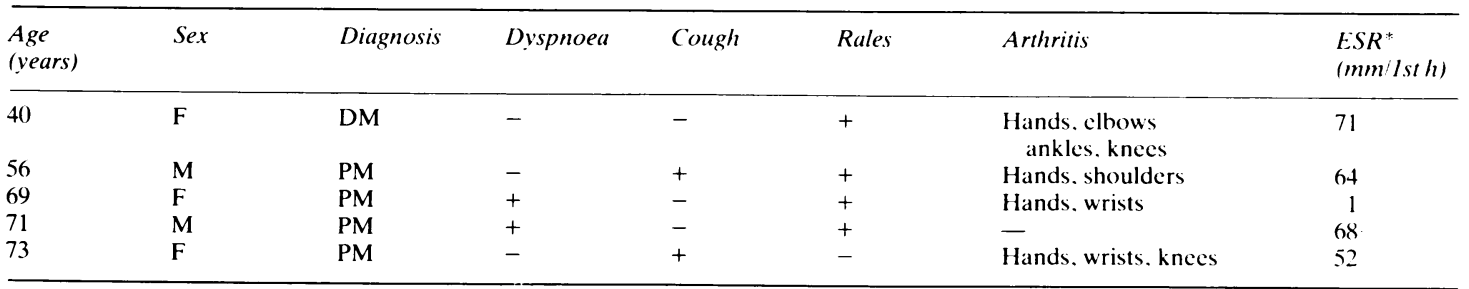

${ }^{*} \mathrm{ESR}=$ erythrocyte sedimentation rate. 

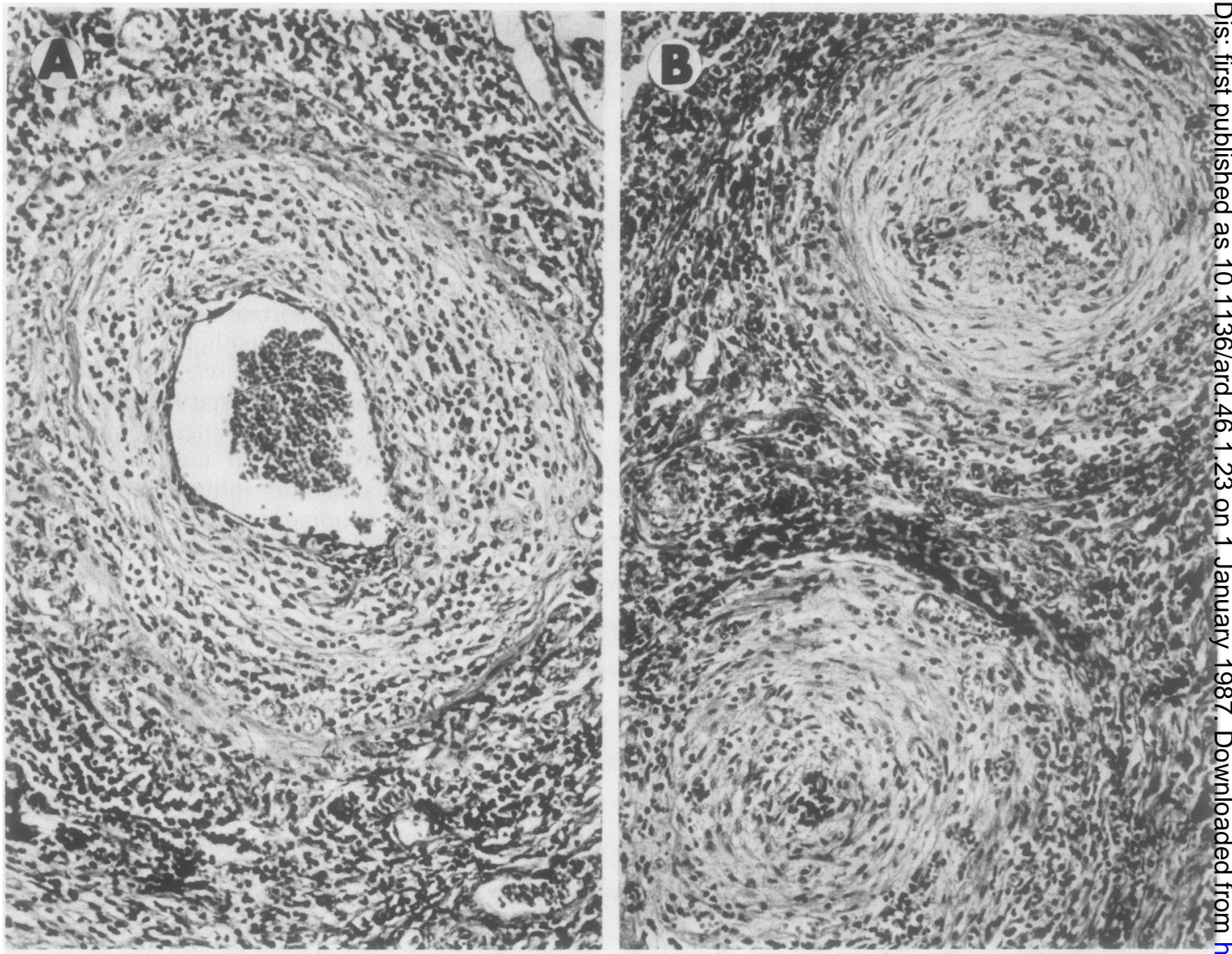

Fig. 2 Two histopathological types of pulmonary vasculitis in PM/DM: $(A)$ active necrotising vasculitis; $(B)$ chronic proliferative healed vasculitis; in both the cellular infiltrate is predominantly lymphomononuclear and plasmacytic. (Haematoxylin and eosin).

and rheumatoid lung disease. The vasculopathy included active necrotising vasculitis and chronic or healed proliferative lesions (Fig. 2). The mean survival of the four patients with PM and pulmonary vasculitis was 12 months after disease onset, while that for the patient with DM was 72 months. Pulmonary artery atherosclerosis was found in two patients, one each with PM and DM, who had been treated with prednisone for 12 years and 14 years respectively.

\section{Infective pneumonitis}

Bronchopneumonia was found at autopsy in 35 cases (PM 13, DM 22). Of these, four had not received prednisone or other immunosuppressive agents. Of the 54 patients treated with prednisone, $30(56 \%)$ developed pneumonia as compared with four of 11
Table 3 Infectious agents identified in patients with $P M / D M$ and bronchopneumonia

\begin{tabular}{|c|c|c|}
\hline Infectious agents & $\begin{array}{l}P M^{*} \\
(n)\end{array}$ & $\begin{array}{l}D M \\
(n)\end{array}$ \\
\hline
\end{tabular}

Pseudomonas aeruginosa

Cryptococcus neoformans

Staphylococcus aureus

Nocardia asteroides

Candida albicans

Mucormycosis

Pneumocystis carinii

Haemophilus influenzae

Streptococcus pyogenes

Pneumococcus

Aerobacter aerogenes

${ }^{*}$ Two infectious agents were identified each in two patients with PM and one with DM. 
$(36 \%)$ not so treated. A history of dysphagia was present in 19 of these cases (PM 7, DM 12). Of the 30 patients without pneumonia, $15(50 \%)$ had a history of dysphagia. In comparison, $54 \%$ of patients with pneumonia had dysphagia.

Infectious agents were identified in 13 patients with pneumonia (PM 5, DM 8) and were usually opportunistic infections (Table 3). Associated pulmonary abscesses were identified in an additional three cases. Bronchopneumonia was also present in three patients with primary or metastatic lung malignancy.

Aspiration pneumonia was identified in six patients (PM 2, DM 4), and five of these had a history of dysphagia. Bronchopneumonia was also present in another 11 patients (PM 5, DM 6) with a history of dysphagia. Cultures were not done in these cases.

\section{Pulmonary oedema}

Significant pulmonary oedema was recognised in 28 patients (PM 12, DM 16) at autopsy. The majority of these patients had cardiac (PM 7, DM 8) or renal (PM 1, DM 2) disease that could account for pulmonary oedema. Of the 10 patients without cardiac or renal disease, eight (PM 4, DM 4) had associated bronchopneumonia. Five of these 28 patients had haemorrhagic pulmonary oedema (PM 3, DM 2).

\section{Malignancies}

Primary pulmonary malignancy was found in two patients with DM, both of whom had grade 4 undifferentiated large cell carcinoma; only one of them was a smoker. Another patient with DM had metastatic carcinoma of the cervical lymph nodes and right hemidiaphragm and was suspected of having a primary lung malignancy. One patient with DM had adenocarcinoma of the kidney with pulmonary and subpleural metastases, and another had reticulum cell sarcoma of the iliac lymph nodes.

None of the patients with PM had a pulmonary malignancy, but adenocarcinoma of the prostate, adenocarcinoma of the adrenal gland, and undifferentiated grade 4 carcinoma of the pancreatic tail were found in one patient each at autopsy.

\section{Diffuse alveolar damage}

Prominent hyaline membrane disease type diffuse alveolar damage, characteristic of adult respiratory distress syndrome, was seen in nine patients with DM and one with PM. These patients had clinically developed the respiratory distress syndrome during the terminal course of their disease.

\section{Pleural disease}

Fibrinous pleuritis was noted in eight patients, four each with PM and DM. Hydrothorax was present in 16 patients (PM 3, DM 13), 15 of these had pulmonary oedema/heart failure. One patient with DM and pleural effusion had primary lung cancer, but the pleural fluid was negative for malignant cells.

\section{Other pulmonary findings}

Pulmonary emboli/infarct were seen in four patients with PM and four with DM. Three of these were bone marrow/fat emboli and probably related to resuscitative efforts; one patient had a tumour embolus.

Emphysematous changes were found in three patients with PM and two with DM, bronchiectasis in two with PM and one with DM, and chronic bronchitis in two patients with DM. Three patients (PM 1, DM 2) had atrophy of the diaphragm.

\section{Discussion}

We have analysed the pulmonary pathological findings in 65 patients with PM/DM seen at the Mayo Clinic, representing the largest series of autopsy cases from a single institution. This is a highly selected group but does show the wide spectrum of pulmonary disease in patients with PM/DM. Some patients with $\mathrm{PM} / \mathrm{DM}$ and underlying pulmonary disease were clinically asymptomatic. The onset of pulmonary disease in these cases was an ominous sign, indicating reduced life expectancy. No routine laboratory test could be relied upon to predict the nature of pulmonary involvement. Chest roentgenograms were fairly reliable in detecting ILD, bronchopneumonia, and pulmonary oedema, but each specific disease could not necessarily be distinguished on the basis of the chest roentgenogram alone.

ILD is the most frequent pulmonary pathological finding recognised to be associated with PM/ DM. ${ }^{1 P}{ }^{15}$ The lung biopsies were corroborated by autopsy findings for the diagnosis of ILD among the small number of patients in whom a biopsy was performed. Almost half of the patients with ILD had associated arthritis as compared with less than a quarter of patients without ILD. The mean survival was reduced in the patients with ILD as compared with those without. None of the patients with ILD had received drugs with potential for significant pulmonary toxicity.

A small group of patients $(5 / 65$, or $8 \%)$ with pulmonary vasculitis was identified in our autopsy series. Pulmonary symptoms and arthritis were each present in $80 \%$ of these cases. All of these patients also had associated ILD. In addition, the patients with vasculitis had high ESR. Thus there may be a subgroup of patients with PM/DM and 
pulmonary symptoms, associated with arthritis and a high ESR, who have underlying pulmonary vasculitis. One of our patients with pulmonary vasculopathy actually presented with pulmonary hypertension which was rapidly fatal. ${ }^{18}$

The reported incidence of pulmonary disease in $\mathrm{PM} / \mathrm{DM}$ varies greatly. In the series reported by Frazier and Miller 5\% of 213 cases had diffuse ILD, ${ }^{4}$ whereas none of the 97 patients reviewed by Bohan et al had pulmonary disease. ${ }^{19}$ In a much smaller series Songcharoen et al found ILD in seven of 15 patients with PM/DM. ${ }^{13}$ Data collected by Dickey and Myers show a $10 \%$ prevalence of lung disease in PM/DM by radiographic evidence and a $31 \%$ prevalence by physiological criteria. ${ }^{5}$

Bronchopneumonia was present in over half the patients who had been treated with corticosteroids. Fifty-six per cent of patients who had received corticosteroids developed bronchopneumonia as compared with $36 \%$ of those who did not. It is possible that the use of immunosuppressive agents predisposes to development of pneumonia. These patients require therapy with immunosuppressive drugs for control not only of $\mathrm{PM} / \mathrm{DM}$ but also for associated ILD. ${ }^{9-12}$ Because of oesophageal dysfunction many of these patients tend to aspirate and develop aspiration pneumonia which may become infective, especially in view of the patients' suppressed immune status. ${ }^{20-22}$ A history of dysphagia was present with equal prevalence, however, in cases with bronchopneumonia and those without. It is conceivable that some patients with bronchopneumonia may have had incipient dysphagia and did not complain of it. A definite diagnosis of aspiration pneumonia was made in six patients at autopsy, five of whom had a history of dysphagia.

Pulmonary oedema was found in $43 \%$ of the cases. Underlying cardiac or renal disease that may have been responsible for pulmonary oedema was present in $64 \%$ of these cases. The prevalence of cardiac involvement in polymyositis has been previously reported..$^{23}$ Of the 10 patients with pulmonary oedema in the absence of underlying cardiac or renal disease, eight had associated bronchopneumonia. It is difficult to be certain whether pulmonary oedema in these latter cases was related to pulmonary infections or was just an agonal event.

Only two patients had a primary pulmonary malignancy. A variety of other malignancies were seen. The prevalence of malignancy, including those in the past, was $15 \%$ and is not significantly higher than that expected in the general population of patients seen at the Mayo Clinic. ${ }^{24}$

Pulmonary involvement is an important cause of morbidity and mortality in PM/DM. The spectrum of pulmonary disorder in these diseases is wider thas? previously recognised. The occurrence of pulmon ary vasculitis in PM/DM is not widely appreciated and, with two exceptions, ${ }^{18}{ }^{25}$ has not been pree viously recorded.

\section{References}

1 Hunninghake G W. Fauci A S. Pulmonary involvement in t) collagen vascular diseases. Am Rev Respir Dis 1979; 11\% 471-503.

2 Mills E S, Matthews W H. Interstitial pneumonitis in der matomyositis. JAMA 1956; 160: 1467-70.

3 Hepper N G G. Ferguson R H, Howard F M Jr. Three types pulmonary involvement in polymyositis. Med Clin North $A \overline{\mathcal{B}}$ 1964; 48: $1031-42$.

4 Frazier A R, Miller R D. Interstitial pneumonitis in associatiơ with polymyositis and dermatomyositis. Chest 1974; 65: 403-7.

5 Dickey B F. Myers A R. Pulmonary disease in polymyosit 6 S dermatomyositis. Semin Arthritis Rheum 1984; 14: 60-76.

6 Young R H, Mark G J. Pulmonary vascular changes iD scleroderma. Am J Med 1978; 64: 998-1004.

7 Miller L R, Greenberg S D. McLarty J W. Lupus lung. Che 1985; 88: 265-9.

8 Bohan A, Peter JB. Polymyositis and dermatomyositis. $N$ En J Med 1975; 292: 344-7, 403-7.

9 Weaver A L, Brundage B M, Nelson R A. Bischoff M B. Pulmonary involvement in polymyositis: report of a case wif response to corticosteroid therapy. Arthritis Rheum 1968: 19v 765-73.

10 Webb D R, Curric G D. Pulmonary fibrosis masking polym ositis. Remission with corticosteroid therapy. JAMA 1972; 22 1146-9.

11 Duncan P E, Griffin J P. Garcia A, Kaplan S B. Fibrosin alveolitis in polymyositis. A review of histologically confirmo cases. Am J Med 1974; 57: 621-6.

12 Schwarz M I, Matthay R A, Salm S A, Stanford R Marmorstein B L. Scheinhorn D J. Interstitial lung disease polymyositis and. dermatomyositis: analysis of six cases and review of the literature. Medicine (Baltimore) 1976; 55: 89-107

13 Songcharoen S. Raju S F. Pennebaker J B. Interstitial luRg disease in polymyositis and dermatomyositis. $J$ Rheumatol 1980 7: 353-60.

14 Salmeron G, Greenberg S D, Lidsky M D. Polymyositis a diffuse interstitial lung disease: a review of the pulmonam histophathologic findings. Arch Intern Med 1981; 141: 1005- 荝.

15 Crystal R G, Gadek J E, Ferrans V J, Fulmer J D, Line B Q Hunninghake G W. Interstitial lung disease: current concepts pathogenesis, staging and therapy. Am J Med 1981; 70: 542-68

16 Crystal R G, Bitterman P B, Rennard S I, Hence A J, Keogh B A. Interstitial lung diseases of unknown cause: disordes characterized by chronic inflammation of the lower respirato: tract. $N$ Engl J Med 1984; 310: 154-66.

17 Basset F, Ferrans V J, Solar P, Takemura T, Fukuda Y. Crysto R G. Intraluminal fibrosis in interstitial lung disease. $A m_{\mathrm{N}} \mathrm{J}$ Pathol 1986; 122: 443-61.

18 Bunch T W, Tancredi R G, Lie J T. Pulmonary hypertension polymyositis. Chest 1981; 79: 105-7.

19 Bohan A, Peter J B, Bowman R L, Pearson C M. computer-assisted analysis of 153 patients with polymyositis de dermatomyositis. Medicine (Baltimore) 1977; 56: 255-86. $\bar{\varnothing}$

20 Venables P J W, Mumford P A, Maini R N. Antibodies nuclear antigens in polymyositis: relationship to autoimmunt 'overlap syndromes' and carcinoma. Ann Rheum Dis 1981; 40 : 217-23.

21 Reichlin M, Maddison P J, Targoff I, et al. Antibodies to nuclear/nucleolar antigen in patients with polymyositis overi syndrome. J Clin Immunol 1984; 4: 40-4.

22 Jansen H M. Shutte A J M. Elema J D. et al. Local immorac 
complexes and inflammatory response in patients with chronic interstitial pulmonary disorders associated with collagen vascular disorders. Clin Exp Immunol 1984; 56: 311-20.

23 Denbow C E, Lie J T, Tancredi R G, Bunch T W. Cardiac involvement in polymyositis. A clinicopathologic study of 20 autopsied patients. Arthritis Rheum 1979; 22: 1088-92.
24 Lakhanpal S, Bunch T W. Ilstrup D M. Melton L J. III. Polymyositis/dermatomyositis and malignancy: Does an association exist? Mayo Clin Proc 1986; 61: 645-53.

25 Pace W R Jr. Decker J L. Martin C J. Polymyositis: report of two cases with pulmonary function studies suggestive of progressive systemic sclerosis. Am J Med Sci 1963; 245: 322-32. 